\title{
Modeling the Motion and Spread of Air Pockets within Stormwater Sewers
}

\author{
Thomas M. Hatcher, Carmen D. Chosie and José G. Vasconcelos
}

\begin{abstract}
There are important adverse effects linked to the presence of entrapped air pockets in stormwater systems. These effects include loss of conveyance, surging caused by air compression, loss of storage, and geysering. The capability to monitor the formation and motion of entrapped air pockets is highly desirable when modeling extreme rain events in stormwater systems. Experimental investigations conducted at Auburn University have led to a better understanding of important flow features related to the motion of entrapped air in closed conduit flows. Results from these studies, which are summarized in this work, have supported the development of an innovative approach to perform simulations of the air pocket motion based on Benjamin's (1968) work on air cavity motion. The proposed model accounts for surface tension in a similar manner to Wilkinson (1982) but incorporates background flows and circular cross-sections. Air motion is described with a non-Boussinesq integral gravity current model approach, assuming a steady state flow for the water. Measured and predicted values for the air pocket leading edge coordinate and celerity are compared. Results indicate that the proposed model is fairly accurate and may constitute an efficient model to describe entrapped air pocket kinematics in closed conduit flows.
\end{abstract}

Keywords: Entrapped air pockets, stormwater sewers, integral model, experimental investigation.

\section{Introduction}

Intense rain events in urban areas lead to large amounts of surface runoff, which is eventually conveyed into stormwater collection systems. Most of the time this leads to the rapid filling of closed conduits, such as sewers, in a highly unsteady fashion that can lead to the entrapment of large air pockets ( $\mathrm{Li}$ and McCorquodale 1999; Zhou, et al. 2002; Vasconcelos and Wright 2006). Issues that have been linked to the presence of air in stormwater systems include increased pressure surges, loss of storage capacity, and geysering. Such issues have led to investigations aimed at the identification of interactions between entrapped air pockets within unsteady water flows.

There are at least three different types of studies linked to the behavior of entrapped air pockets. The first type approaches the problem of air pocket interactions in the perspective of stormwater systems and led to the development of numerical models and experimental investigations that explore some of these interactions. In these studies air pockets are linked to flow regime transition episodes (either abrupt or gradual), and one concern is the pressurization of air. One of the first investigations on this topic was presented by Martin (1976) for the compression of an air pocket at the dead end of an upward inclined pipe upon the sudden opening of a valve connecting the pipeline to a high head reservoir. This study demonstrated that air pressure heads can exceed by many times the pressure head of the reservoir that generated the flow in the pipeline. In addition, the smaller air pockets lead to the largest air pressure surges.

The entrapment of air pockets during the rapid filling of closed conduits was the focus of the works of Haman and McCorquodale (1982) and Li and McCorquodale (1999). The shear forces caused by the relative motion of air and water led to the development of waves in the conduit that eventually touched the pipe crown, leading to pocket entrapment and flow pressurization. Zhou et al. (2002) presented experimental and numerical results of a rapid filling pipe with air pocket compression that is allowed to ventilate during the filling process. The authors found that rapid filling with small ventilation orifices led to pressure hydrographs that are qualitatively similar to Martin (1976); larger orifices led to small air pressurization and waterhammer-like pressures upon the impact of the inflows into the ventilation orifices, much in the same way as an air slam event (Lingireddy et al. 2004). Vasconcelos and Leite (2012) performed studies in which air pockets of various volumes were suddenly entrapped in an initially steady flow by means of valve maneuvering. The authors found that pressure surge results are significantly altered when pressure relief is provided during the cycles of air compression and expansion.

Other research investigations focused on the mechanisms of air-triggered geysering events. Nielsen and Davis (2009) and Wright et al. (2011) presented studies indicating that the sudden release of air through partially filled vertical shafts can cause dramatic geyser episodes. This is in agreement with a study by

Hatcher, T.M., C.D. Chosie and J. Vasconcelos. 2014. "Modeling the Motion and Spread of Air Pockets within Stormwater Sewers." Journal of Water Management Modeling C370. doi: 10.14796/JWMM.C370.

(c) CHI 2014 www.chijournal.org ISSN: 2292-6062 
Vasconcelos and Wright (2011) in which this event was reproduced in a laboratory. A numerical model was proposed that was able to accurately reproduce experimental measurements.

Modeling the rapid filling of closed conduit flows accounting for air pockets is a relatively new research area, and there has been some initial progress in accounting for air-water interactions during filling events. Vasconcelos and Wright (2009) were able to simulate rapid filling with a Saint-Venant equation model modified with the TPA method (Vasconcelos, et al. 2006). Air pressurization was incorporated in the model, which was able to reproduce the experimental measurements reasonably well. Experiments conducted by Trindade and Vasconcelos (2013) showed good agreement with modeled results attained from a new pipeline filling model that simulates the air phase and outflow using the isothermal Euler equations. A key limitation of all existing studies is that processes that would naturally lead to air pocket motion and spreading, which are linked to the differences in densities between air and water, are not accounted for in the analysis.

The second type of study related to air motion in closed conduits has focused more on the fundamental aspects of the flow dynamics. These studies dealt with fundamental aspects of the air motion such as the advance of an air cavity or finite length air pocket from the open end of a pipe. Such flow conditions are referred to as gravity currents and are created from density differences between two fluids. Gravity currents are exemplified in avalanches, sand storms and lock exchange flows in channels, amongst many other phenomena. In broad terms one refers to Boussinesq currents when the density differences between fluids are small, e.g. $<20 \%$. Non-Boussinesq currents are those flows occurring when density differences are very large as in the spreading of an air pocket along the crown of a pipe filled with water. One of the earliest works on gravity currents was by Benjamin (1968). Benjamin proposed a steady state theory to describe such air cavity motion when the air was allowed to flow freely into a tube. Benjamin determined the possible air cavity depths $(y / H \leq 0.5$, in which $y$ is the depth of the air cavity and $H$ is the height of the tube) and concluded that dissipation existed for depth ratios $y / H<0.5$.

In an attempt to quantify the effects of surface tension and unsteadiness, which were not considered by Benjamin, Wilkinson (1982) conducted experiments in rectangular tubes filled with water in which one end was opened to admit air. One of the primary objectives was to describe the front speeds and shapes of the resulting air cavities when the air inflow was throttled. In his experiments the energy conserving steady state solution $y / H=0.5$ existed when the air was allowed to flow freely out of one end of the tube. Surface tension and wall boundary effects were negligible in such conditions. For throttled flows in which one end of the tube was partially opened, Wilkinson (1982) determined that the flow was unsteady when the depth ratio at the outlet was $0.22 \leq y / H<0.5$. In this scenario the speed and shape of the air cavity front was the same as for energy conserving flows, but the front was followed by a hydraulic jump moving at a slower speed than the front. When the air cavity depth ratio $y / H<0.22$ the flow was again steady but corrections for surface tension were recommended. For this steady state scenario, which was predominant for the experiments conducted in this work, Wilkinson (1982) obtained an analytical solution. One of the drawbacks of this approach is that the expression represents one equation for two unknowns: air pocket front velocity and depth.

Baines et al. (1985) performed similar experiments except that a constant volume of air was released at one end of a rectangular tube. Three phases were observed in the experiments: the velocity and depth of the front were steady (phase 1); the front speed decreased monotonically in which there was a balance between inertia and drag (phase 2); the front erratically stopped and started before coming to rest (phase 3 ). The characteristics of phase 1 were generated a few tenths of a second after the initial gate release and are dependent on the initial depth ratio $y_{0} / H$ (where $y_{0}$ is the initial air pocket depth measured from the pipe centerline). For $0.7 \leq y_{0} / H \leq 1$, Benjamin's energy conserving theory developed. A different steady state front was observed by the authors for smaller depth ratios in which the flow upstream of the nose resembled a shallow neck. In both instances a shock developed that, once reflected off of the end wall, eventually overtook the front. This instance generates the beginning of phase 2 (the kinematics of phase 2 are independent of the initial depth ratio). This feature was also demonstrated in Boussinesq gravity currents by Rottman and Simpson (1983).

More recently studies were focused on the motion of discrete pockets in closed conduits. The work of Glauser and Wickenhauser (2009) included a study of the motion of air pockets in a downward inclined experimental apparatus. The authors observed the existence of a critical air pocket volume for a certain water flow rate and slope. Below this volume threshold the pocket would be dragged by the flow against buoyancy, but this volume also depended on the pipeline slope as it affected the shape of the air pocket. However, in their study, the tested slopes included only values between $1.7 \%$ and $8.7 \%$, much higher than those generally encountered in stormwater storage tunnels. Moreover, the slopes tested were only favourable (when drag caused by the water flow opposes air pocket buoyancy), and thus expressions for the air pocket involving the combination of these two forces were not presented. Vasconcelos and Chosie (2012) expanded these studies to include favourable slopes and shallower slopes that are more commonly found in stormwater systems. For favourable slopes, the authors stated that the air pocket front velocity is approximated by the summation of the background flow and the pocket celerity in quiescent conditions (similar to the conclusions of Hallworth et al. [1998] for Boussinesq flows). The air pocket celerity is a function of the pocket's thickness and is further discussed in the Methodology section.

Finally, a third type of investigation has focused on studying the conditions that are linked to the hydraulic removal of air in closed conduits, with clear applications for pipeline flows. 
Various formulas (Falvey 1980; Pozos et al. 2010; Pothof and Clemens 2010) were proposed to predict the minimum average velocity that clear air pockets from pipelines based on air pocket dimension, pipeline slopes, and such. Albeit relevant, these studies do not attempt to calculate the interactions of air-water in terms of pressure surges; nor do they calculate the motion or spreading of air due to the differences in fluid densities, and thus are not relevant to the objectives of the present work.

There is an important knowledge gap that stands at this point, which is linked to the inability to account for both the spreading and the pressurization of air pockets during simulations of rapid filling events. A standing challenge in this task is the inability of Saint-Venant equations to represent flow conditions at the edge of air pockets where free surface flows are curved and pressures are non-hydrostatic. A new modeling formulation that avoids the limitations of the Saint-Venant equations is needed, and is presented in this work.

\section{Objectives}

The objective of this work is to present a new formulation that is based on the integral model approach utilized for gravity currenty flows. This relatively simple approach avoids the modeling difficulties of the Saint-Venant equations to represent the curved air-water front. This work assesses the effectiveness of this approach by comparison with the experimental results on the motion of entrapped air pockets presented by Vasconcelos and Chosie (2012). The proposed model was tested for all of the air pocket propagation results collected in their experiments for horizontal slopes, with and without ambient flows, including the initial slumping and self-similar flow stages.

\section{Methodology}

The simulation of gravity current flows may be performed with three primary methods which are employed according to the objectives of the modeling effort. The solution of the full NavierStokes equations accounting for multiphase flows through CFD models is the most sophisticated and time consuming and is applicable in cases when flow details and mixing process are of interest. A second alternative involves using the shallow water equations, which is a good option when the simulation is focused on the representation of internal waves or the gravity current front propagation. The third and simplest approach is the integral model, which neglects changes in the gravity current thickness and uses a relationship between the gravity current thickness and its propagation speed to update the location of the gravity current front. Integral models are also referred to as box models (Ungarish 2009) and have been validated by studies such as the one presented by Harris et al. (2001).

The governing equations for the proposed integral model consist of a front condition and a continuity equation (Ungarish 2009). The front condition is based on the steady state analysis from Benjamin (1968) in circular pipes and relates the thickness of the gravity current to its propagation speed. Accounting for ambient crossflows, the front condition for a gravity current propagating in a rectangular channel is provided in Hallworth et al. (1998). For the following integral model formulation, this expression for the front celerity $u_{f}$ has been adapted to circular cross-sections:

$$
u_{f}=\frac{d x_{f}}{d t}=\operatorname{Fr} \sqrt{g D}+U
$$

where:

$$
\begin{aligned}
u_{f}= & \text { distance traveled by the respective cavity front }, \\
t= & \text { time } \\
F r= & \text { local Froude number at the upstream or } \\
& \text { downstream air pocket leading edge, } \\
D= & \text { pipe diameter, and } \\
U= & \text { background flow velocity. }
\end{aligned}
$$

To determine the expression for Fr, Benjamin's analysis was utilized with the addition of surface tension effects. In a frame of reference moving with the air pocket front, the flow forces upstream of the front and below the air pocket were set equal to one another (Benjamin 1968). The cavity Froude number, $u_{f} \sqrt{ }(g D)$, was isolated using Bernoulli's expression with algebraic manipulation. After integrating and solving for $x_{f}$, Equation 1 is transformed into the proposed integral model:

$$
\begin{aligned}
& x_{f}=(F r \sqrt{g D}+U)\left(t_{\text {new }}-t_{\text {old }}\right) \\
& F r=\left[\frac{\pi D^{2} A_{2}-\frac{2 \pi D A_{2} \sigma}{\rho g r_{c}}-4 A_{2}\left(A_{2} \cos \alpha+\frac{1}{6} D^{2} \sin ^{3} \alpha\right)}{\pi D^{2}\left(\frac{1}{2} \pi D^{2}-A_{2}\right)}\right]^{\frac{1}{2}}
\end{aligned}
$$

where:

$$
\begin{aligned}
t_{\text {new }}= & \text { updated time parameter, } \\
t_{\text {old }}= & \text { previous time parameter, } \\
a= & \text { pipe half-angle ( } 2 a \text { is the angle subtended from } \\
& \text { the center of the pipe to the free surface }), \\
\sigma= & \text { surface tension, } \\
r_{c}= & \text { radius of curvature, and } \\
A_{2}= & \text { cross-sectional area below air pocket } \\
& =(\pi-a+1 / 2 \sin a) D^{2} / 4 .
\end{aligned}
$$

Since there are initially two air intrusion fronts (because an air pocket propagation or spreading can occur in two directions along the pipeline), Equation 2 is solved twice at each time step (to compute $x_{f u}$ and $x_{f D}$ ). The methodology used to solve each front location is identical except that the sign of $U$ changes depending on the direction of the front motion and background flow. One of the difficulties in solving Equation 2 is that $x_{f}\left(x_{f u}\right.$ or $\left.x_{f D}\right)$ is a function of $a$ and vice versa. For the implementation utilized in this work, $x_{f}$ was first computed from parameters determined at the previous time step; then $a$ was updated using the following volume-continuity expression:

$$
V o l=\frac{L D^{2}}{4}\left(\alpha-\frac{1}{2} \sin 2 \alpha\right)
$$


where:

$$
\begin{aligned}
V o l & =\text { air pocket volume, and } \\
L & =\text { air pocket length }\left(=x_{0}+x_{f U}+x_{f D}\right)
\end{aligned}
$$

This nonlinear expression was solved using the NewtonRaphson method with a precision of $10^{-5}$. This iterative process was repeated until the change in $a$ was less than the specified precision (i.e. $10^{-5}$ ) before moving on to the next time step. $A$ schematic diagram for this integral model formulation is displayed in Figure 1 where $x_{0}$ and $y_{0}$ represent the initial air pocket length and depth respectively. One needs to consider that the geometry of the pipe is $3 \mathrm{D}$ and that $y$ and $y_{0}$ are measured from the pipe centerline.
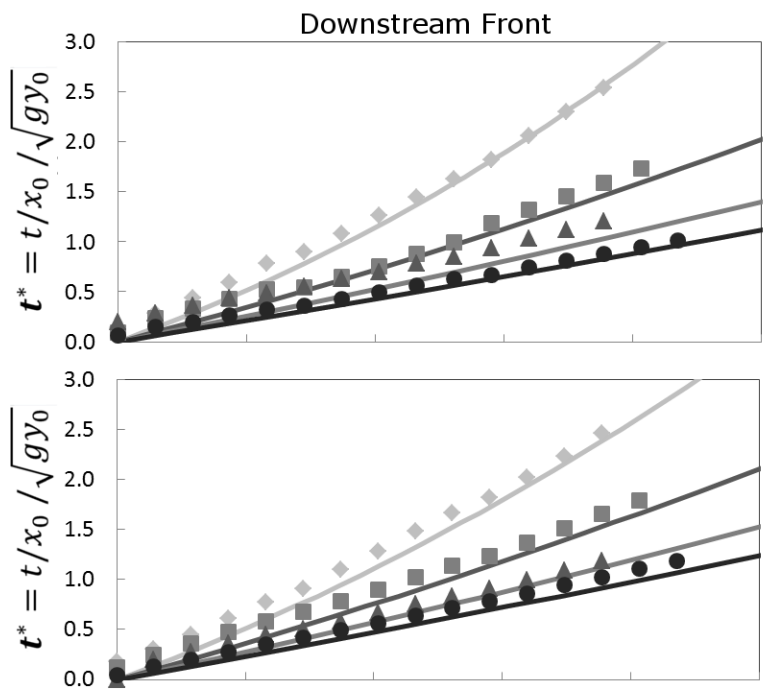

a)

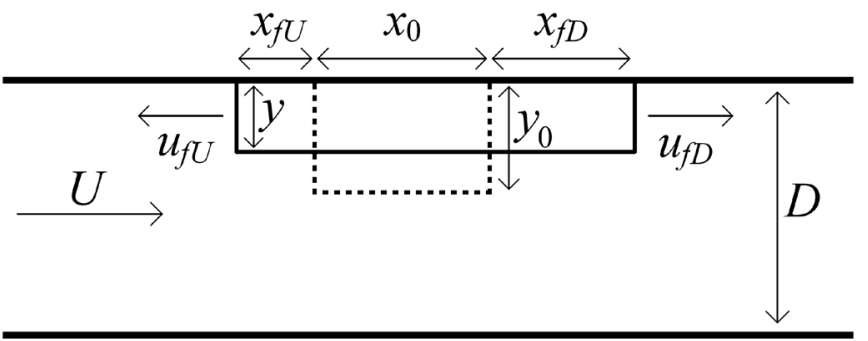

Figure 1 Schematic diagram of an air pocket propagating in a closed conduit (2D view) using the box model assumption (the direction of $u_{f D}$ is always positive while the sign of $u_{f U}$ will vary with $U$ and $y / D$; the subscripts $f U$ and $f D$ denote updated variables (pocket length $x$ or celerity $\left.u_{f}\right)$ at the current time step.
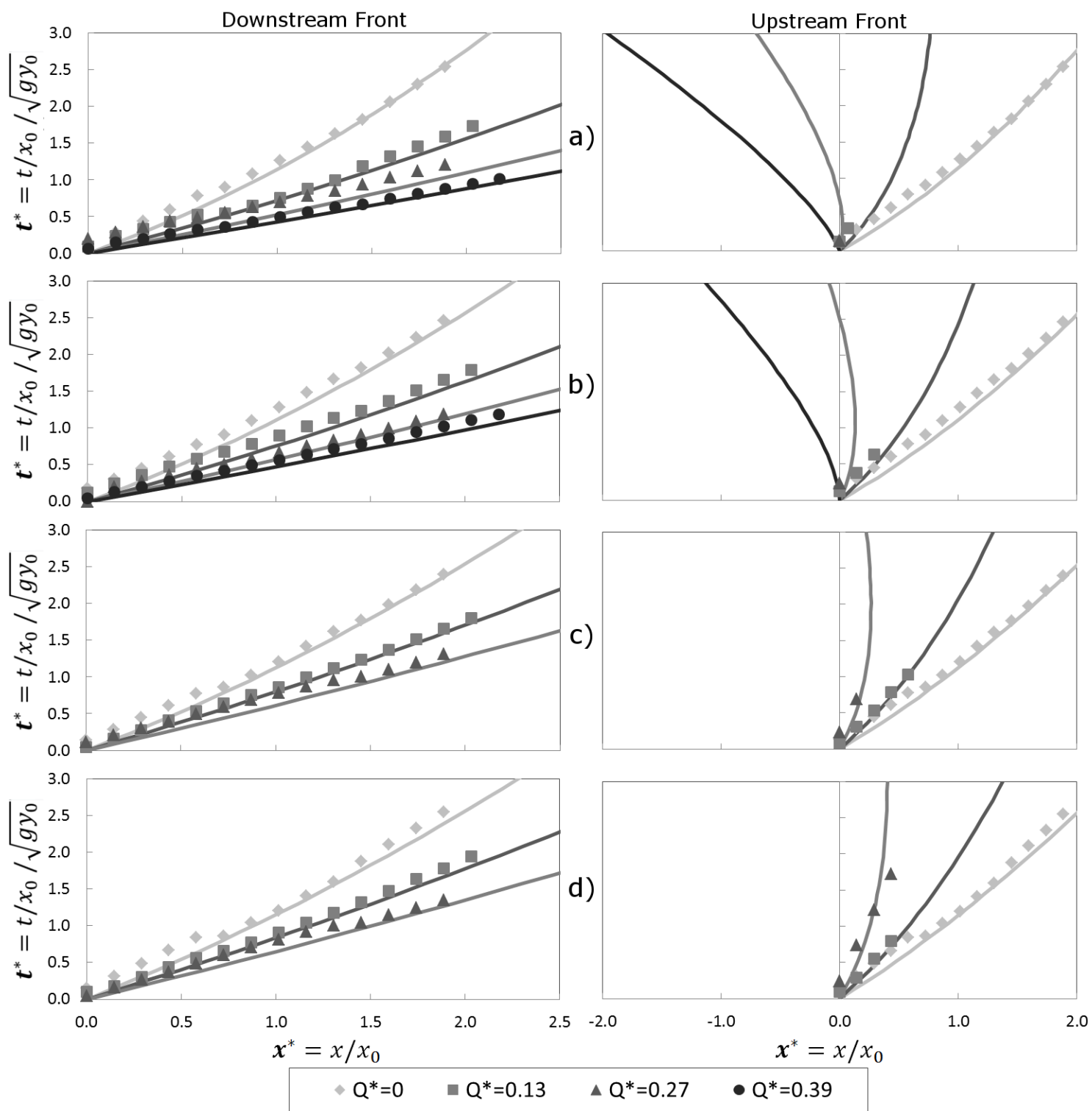

Figure 2 Air front trajectory comparison between experiments and the proposed integral model for various background flows and pocket volumes ( $\mathrm{a}, \mathrm{b}, \mathrm{c}$ and $\mathrm{d}$ ); the lines represent the integral model, and the color indicates the respective background flow. 
The integral model formulated in this work accounts for buoyancy, drag, surface tension and background flows, but there are important limitations. The model formulation utilizes steady state theory for the air intrusion and assumes no displacement for the stagnation point. The steady state assumption is valid during the initial slumping stage, but the air pocket motion will diverge from steady state theory in later stages of the propagation (Simpson 1997). In addition, the spatial variation in depth is neglected, so the air-water interface is assumed to be horizontal. For the surface tension parameters, $\sigma\left(0.081 \mathrm{~kg} / \mathrm{s}^{2}\right)$ and $r_{c}(0.0034 \mathrm{~m})$ were implemented from recommendations in Wilkinson (1982) for rectangular pipes. The applicability of these values to circular pipes will be discussed in the Results section, together with other modeling assumptions.

The numerical model results were compared to experimental measurements presented by Vasconcelos and Chosie (2012). The range of conditions selected for comparison is presented in Table 1. In total fourteen different conditions were included in this comparison.

Table 1 Range of experimental conditions tested in numerical modeling studies.

\begin{tabular}{|c|c|}
\hline Parameter & Range Tested \\
\hline Flow rate $Q^{*}=Q / \sqrt{ }\left(g D^{5}\right)$ & Up to four values ranging from $Q^{*}=0$ to $Q^{*}=0.388$ \\
\hline Pipeline slope & Horizontal \\
\hline Air pocket volume (normalized by $D^{3}$ ) & Four values in the range $\mathrm{Vol}^{*}=1.27$ to $\mathrm{Vol}^{*}=4.13$ \\
\hline
\end{tabular}

\section{Results and Discussion}

The modeling results are presented in dimensionless units throughout this section. Except for the changes caused by boundary and surface tension effects, the dimensionless results converge for air intrusions with equivalent depth ratios $\left(y_{0} / D\right)$ and background flows (see Figure 1 for reference). Values for length, depth, velocity, flow rate and time values are normalized by $x_{0^{\prime}} y_{0^{\prime}}$ $\sqrt{ }(g D), \sqrt{ }\left(g D^{5}\right)$ and $x_{0} / \sqrt{ }(g D)$ respectively unless otherwise stated.

\subsection{Air Pocket Front Trajectory}

The trajectory of both upstream and downstream air fronts determined from experiments is compared to the proposed integral model predictions in Figure 2 . The surface tension $\left(0.081 \mathrm{~kg} \mathrm{~s}^{2}\right)$ and radius of curvature $(0.0034 \mathrm{~m})$ are based on recommendations from Wilkinson (1982). As displayed in Figure 2, the proposed model is fairly accurate for all conditions tested. The consistency between the numerical model and experiments suggests the surface tension parameters are fairly accurate for both rectangular and circular pipe cross-sections.

For the upstream air front, there are not as many experimental results due to video camera limitations and the difficulty in measuring this front. When the front moved downstream of the initial location, the results were not recorded as there was not a camera at this location. In addition, larger background flows caused the upstream front to break apart making data recording difficult. For the experimental results presented in Figure 2, the integral model compares well.

The proposed numerical model was also tested for larger pipe diameters (>1 m)) to compare the proposed solution to Benjamin's theory. For this scenario, the air pocket velocities converged as expected (the results are not presented). The importance of the surface tension is a function of the relative depth $(y / D)$ and background flow $(U)$. For $Q^{*}=0$ conditions $(D=101.6 \mathrm{~mm})$, surface tension caused the proposed integral model to significantly diverge from Benjamin's theory. This difference between solution strategies increased for smaller air volumes or pocket depths. There was a noticeable but not as significant divergence for $Q^{*}=0.13$ experiments. The integral model converged to Benjamin's energy conserving solution for larger background flows $\left(Q^{*}=0.27\right.$ and $\left.Q^{*}=0.39\right)$.

\subsection{Air Cavity Froude Number}

The air cavity Froude number, defined as $F r=u_{f D} / \sqrt{ }(g D)$, is compared in Table 2 for different background flows and pocket volumes. For $u_{f D}$ an average value was determined from stationary video cameras during the initial slumping stage. The results indicate that the integral model is within $\sim 10 \%$ accuracy for all of the conditions tested. When there is no background flow, the integral model presents the most accurate results. The model also simulates air intrusions with background flows reasonably well (typically $3 \%$ to $9 \%$ accuracy). The upstream air front is not included in this analysis because of the limited amount of experimental data.

Table 2 Cavity Froude number comparison between experiments and proposed integral model for different background flows and pocket volumes at the downstream front.

\begin{tabular}{lcccc}
\hline & $Q^{*}=0$ & $Q^{*}=0.13$ & $Q^{*}=0.27$ & $Q^{*}=0.39$ \\
\hline Experiment & 0.271 & 0.429 & 0.617 & 0.786 \\
Integral Model & 0.268 & 0.467 & 0.664 & 0.826 \\
Error w/exp. (\%) & -1.1 & +8.9 & +7.6 & +5.1 \\
& & & $V^{*}=1.27$ & \\
Experiment & 0.334 & 0.497 & 0.708 & 0.804 \\
Integral Model & 0.341 & 0.537 & 0.728 & 0.896 \\
Error w/exp. (\%) & +2.1 & +8.1 & +2.8 & +11.4 \\
Experiment & & & $V 0 I^{*}=3.18$ & \\
Integral Model & 0.389 & 0.559 & 0.724 & $\mathrm{~N} / \mathrm{A}$ \\
Error w/exp. (\%) & 0.392 & 0.579 & 0.775 & 0.944 \\
& +0.8 & +3.6 & +7.0 & $\mathrm{~N} / \mathrm{A}$ \\
Experiment & & & $\mathrm{Vol} I^{*}=4.13$ & \\
Integral Model & 0.403 & 0.565 & 0.760 & $\mathrm{~N} / \mathrm{A}$ \\
Error w/exp. (\%) & 0.427 & 0.612 & 0.809 & 0.972 \\
\hline & +6.0 & +8.3 & +6.5 & $\mathrm{~N} / \mathrm{A}$ \\
\hline
\end{tabular}

In Figure 3 the cavity Froude number is analyzed for different fractional depths. For each background flow, a near linear pattern emerges for the cavity in which the slope decreases as 
the fractional depth increases. For larger background flows (e.g. $Q^{*}=0.39$ ), the integrity of the front is compromised due to larger shear stresses, and it was difficult to obtain accurate measurements at the leading edge. For the conditions tested in this paper, the cavity Fr ranged from 0.271 to 0.403 depending on the initial depth ratio in the absence of background flow. These magnitudes are significantly smaller than the maximum Fr that can exist.

When air is allowed to flow freely into one end of a horizontal tube, the cavity $F r$ is 0.542 excluding surface tension and boundary effects (Benjamin 1968).

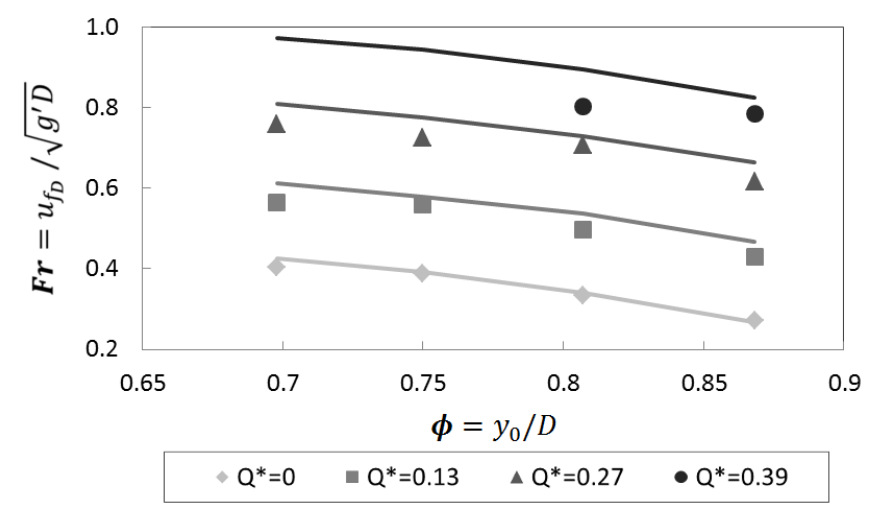

Figure 3 Air cavity Froude numbers for various fractional depths and background flows during the initial slumping stage (the symbols and solid lines denote experimental measurements and integral model predictions, respectively; the colors represent the respective background flows).

\subsection{Relative flow velocity}

A comparison between predicted and modeled values for the relative flow velocity $\left(u_{f}-U\right) / \sqrt{ }(g D)$ is presented in Figure 4 for the same range of pocket volumes and background flows presented above. In general, the experimental results show that the initial relative gravity front velocity is zero and rapidly increases with time following the pocket release. The integral model does not incorporate the process of air pocket acceleration, predicting instantaneous non-zero relative flow velocities.

For different background flows, the experimental results display a convergence in this velocity parameter. The relative velocity increases for larger pocket volumes (this range is approximately 0.3 to 0.4 , see Figure 2 ). The integral model presents fairly accurate results compared to experimental observations. Depending on the air pocket volume, the integral model underestimates the relative flow velocity by $10 \%$ to $22 \%$.

\section{Future Work}

The proposed integral model simulated the experimental results very well and was able to describe both upstream and downstream air front motion. One of the novelties in this model was to depart from approaches based on mass and momentum conservation that are usually applied in the description of air-water interfaces. These older approaches can be applied in cases when air is being displaced by a water inflow front much in the same way as a water piston. However, in the case of an entrapped air pocket in which there is not such front, entrapped pockets will

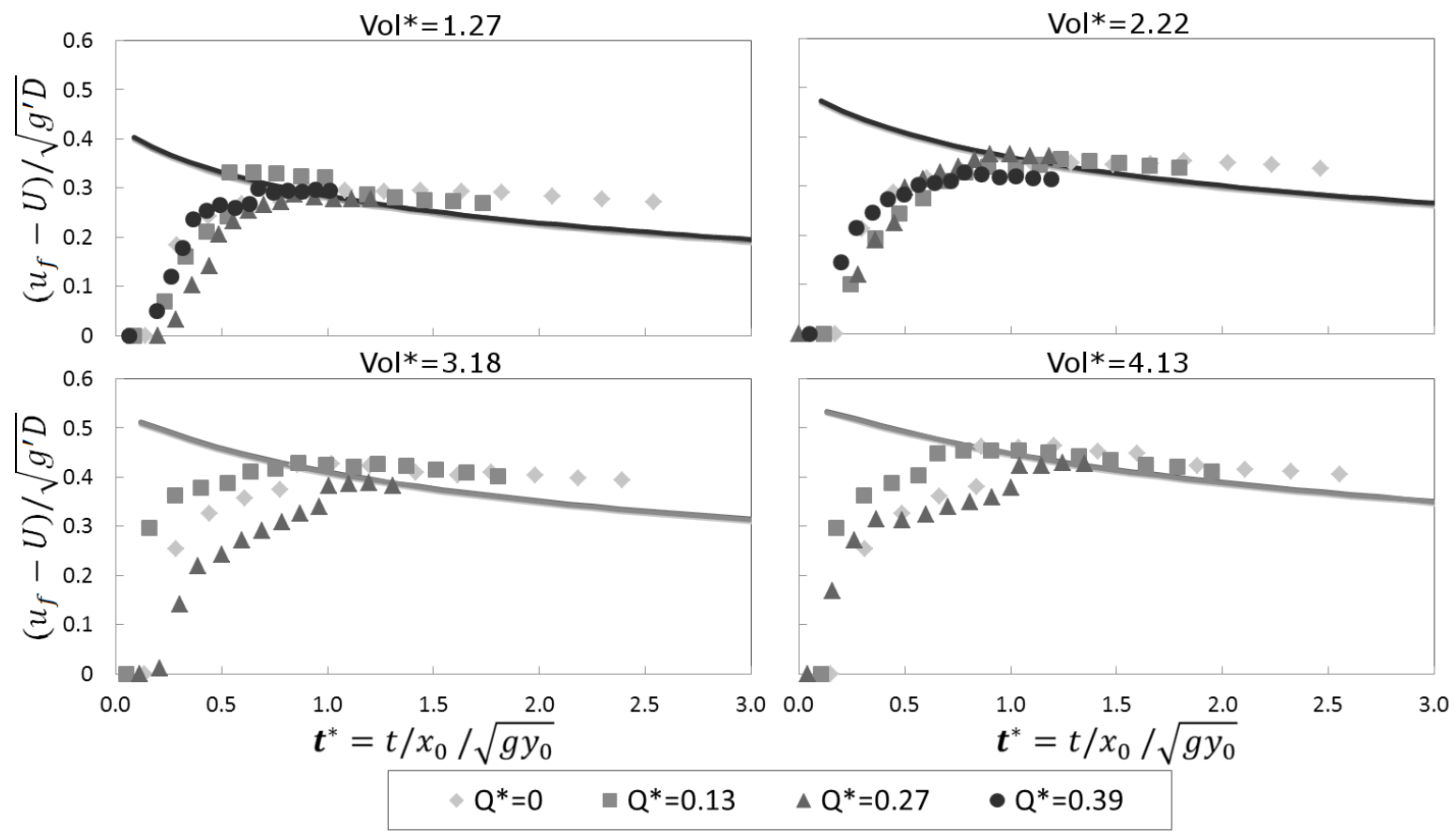

Figure 4 Relative velocity comparison between experiments and proposed integral model for the downstream front; the solid lines represent the integral model, and the color indicates the respective background flows. 
move due to drag forces and buoyancy forces that will cause the pocket to advance into higher points and spread.

This integral model approach, which was implemented in other contexts such as Boussinesq gravity current flows, served as the basis for the model used in this presentation. An important improvement was the inclusion of background flows and surface tension effects in the simulation of air pocket motion within pressurized flows in circular pipes. The results presented here are promising, indicating that such an approach may be coupled with a more sophisticated model to simulate unsteady filling processes in stormwater systems. Due to its simplicity, this method may be integrated into a more complex model to simulate the filling process of stormwater tunnels or other closed conduit systems. The overarching goal is to improve the range of air-water interactions that can be represented in numerical modeling efforts to simulate such systems.

Although the integral model results are promising, more experimental validation is still necessary. The scale effects associated with different pipe diameters are unknown. Also, air pocket fragmentation is not considered in the proposed model, and the impacts of lumping all the air mass in a single pocket to model accuracy is yet unknown. A larger range of experimental conditions is recommended to test this model, such as those conducted in Wilkinson (1982) or Baines et al. (1985) for circular pipes. In future iterations of the proposed integral model, these potential issues will be addressed and effects of slope and unsteady motion will be analysed.

\section{Acknowledgments}

The authors would like to acknowledge the support provided by LimnoTech Inc., and by Auburn University, which have funded part of this research.

\section{References}

Baines, W. D., J. W. Rottman and J. E. Simpson. 1985. "The Motion of Constant-Volume Air Cavities in Long Horizontal Tubes." Journal of Fluid Mechanics 161:313-27.

Benjamin, T. B. 1968. “Gravity Currents and Related Phenomena." Journal of Fluid Mechanics 31:209-48.

Falvey, H. T. 1980. Air-Water Flow in Hydraulic Structures. Engineering Monograph No. 41. Denver: U. S. Department of the Interior, Water and Power Resources Service.

Glauser, S. and M. Wickenhauser. 2009. “Bubble Movement in Downward-Inclined Pipes." Journal of Hydraulic Engineering 135 (11): 1012-5.

Hallworth, M. A., A. J. Hogg and H. E. Huppert. 1998. “Effects of External Flow on Compositional and Particle Gravity Currents." Journal of Fluid Mechanics 359:109-42.

Hamam, M. A. and J. A. McCorquodale. 1982. "Transient Conditions in the Transition from Gravity to Surcharged Sewer Flow." Canadian Journal of Civil Engineering 9:189-96.
Harris, T. C., A. J. Hogg and H. E. Huppert. 2001. "A Mathematical Framework for the Analysis of Particle Driven Gravity Currents." Proceedings of the Royal Society of London A 457:1241-72.

Li, J. and A. McCorquodale. 1999. "Modeling Mixed Flow in Storm Sewers." Journal of Hydraulic Engineering 125 (11): 1170-80.

Lingireddy, S., D. J. Wood and N. Zloczower. 2004. “Pressure Surges in Pipeline Systems Resulting from Air Releases." Journal American Water Works Association 96 (7): 88-94.

Martin, C. S. 1976. “Entrapped Air in Pipelines." In Proceedings of the Second International Conference on Pressure Surges, edited by H. S. Stephens, A. L. King and C. A. Stapleton. Cranfield, Bedfordshire: BHRA.

Nielsen, K. D. and A. L. Davis. 2009. “Air Migration Analysis of the Terror Lake Tunnel." In Proceedings of the 33rd IAHR Congress, Vol. 1, 262-8. Madrid: IAHR (International Association of Hydraulic Engineering and Research)/Curran Associates. ISBN 978-1-61738-231-4.

Pothof, I. and F. Clemens. 2010. “On Elongated Air Pockets in Downward Sloping Pipes." Journal of Hydraulic Research 48 (4): 499-503.

Pozos, O., C. A. Gonzalez, J. Giesecke, W. Marx and E. A. Rodal. 2010. "Air Entrapped in Gravity Pipeline Systems." Journal of Hydraulic Research 48 (3): 338-47.

Rottman, J. W. and J. E. Simpson. 1983. “Gravity Currents Produced by Instantaneous Releases of a Heavy Fluid in a Rectangular Channel." Journal of Fluid Mechanics 135:95-110.

Simpson, J. E. 1997. Gravity Currents in the Environment and the Laboratory. Cambridge: Cambridge University Press.

Trindade, B. C. and J. G. Vasconcelos. 2013. "Modeling of Water Pipeline Filling Events Accounting for Air Phase Interactions." Journal of Hydraulic Engineering 139 (9): 921-34. doi:10.1061/(ASCE)HY.1943-7900.0000757.

Ungarish, M. 2009. An Introduction to Gravity Currents and Intrusions. Boca Raton, FL: Chapman and Hall/CRC. ISBN 9781584889038.

Vasconcelos, J. G. and C. D. Chosie. 2012. “Kinematics of Entrapped Air Pockets Within Stormwater Storage Tunnels." Journal of Water Management Modeling R246-10. doi: 10.14796/JWMM.R246-10.

Vasconcelos, J. G. and G. J. Leite. 2012. "Pressure Surges Following Sudden Air Pocket Entrapment in Stormwater Tunnels." Journal of Hydraulic Engineering 134 (7): 984-92.

Vasconcelos, J. G. and S. J. Wright. 2009. "Investigation of Rapid Filling of Poorly Ventilated Stormwater Storage Tunnels." Journal of Hydraulic Research 47 (5): 547-58.

Vasconcelos, J. G. and S. J. Wright. 2011. "Geysering Generated by Large Air Pockets Released through Water-Filled Ventilation Shafts." Journal of Hydraulic Engineering 137 (1): 112-5. 
Vasconcelos, J. G., S. J. Wright and P. L. Roe. 2006. "Improved Simulation of Flow Regime Transition in Sewers: Two-Component Pressure Approach." Journal of Hydraulic Engineering 132 (6): 553-62.

Wilkinson, D. L. 1982. "Motion of Air Cavities in Long Horizontal Ducts." Journal of Fluid Mechanics 118:109-22.
Wright, S. J., J. W. Lewis and J. G. Vasconcelos. 2011. “Geysering in Rapidly Filling Stormwater Tunnels." Journal of Hydraulic Engineering 137 (5): 543-55.

Zhou, F., F. E. Hicks and P. M. Steffler. 2002. "Transient flow in a rapidly filling horizontal pipe containing trapped air." Journal of Hydraulic Engineering 128 (6): 625-34.

Thomas M. Hatcher Auburn University, Auburn, AL.

Carmen D. Chosie Auburn University, Auburn, AL.

José G. Vasconcelos Auburn University, Auburn, AL. 